\title{
La segmentación de textos. El comportamiento evolutivo entre cinco $y$ once años
}

\section{Rosa Ana Clemente *}

\author{
Universidad de Salamanca
}

$\mathrm{L}$

OS gramáticos organizan los lenguajes naturales separando unidades (las fundamentales varían según las épocas y las teorías lingüísticas), con objeto de estructurar jerárquicamente los elementos participantes en un discurso. Algunas unidades como letra o palabra tienen amplia tradición popular, mientras que otras parecen quedar restringidas para uso de los enierados (fonema, semantema, etcétera).

El conocimiento-aprendizaje de estas unidades se produce en la vida infantil fundamentalmente por dos vías prácticamente simultáneas: en primer lugar el aprendizaje de la versión lecto-escrita del castellano obliga a manejar estos elementos, los arriba calificados como populares serían los más beneficiados por imperativos más o menos convencionales de la ortografía (McCaughey, M., y otros, 1980); en segundo lugar, el aprendizaje de la propia gramática exige del niño en algún momento de la escolaridad obligatoria el conocimiento y el manejo pragmático de estas nociones.

En ambos casos la capacidad cognitiva exigida entraría dentro del ámbito meta-lingüístico, puesto que se pediría al niño la reflexión directa sobre su lengua materna; el castellano se tomaría no como vehículo de comunicación/representación, sino como objeto de estudio en sí mismo. En trabajos con el inglés se ha demostrado que la capacidad metacognitiva de valorar la importancia de las unidades segmentadas de un texto para su comprensión, tiene niveles evolutivos entre ocho y diez años (Brown, A. L., y Smiley, S. S., 1977).

En este trabajo nos limitaremos a indagar sobre el primero de los sistemas de acceso, dejando para una próxima publicación la vía estrictamente gramatical.

* Dirección de la autora: Universidad de Salamanca, Facultad de Filosofía y CC. EE. Departamento úc 
En este caso, el objetivo concreto para el que exponemos los resultados de un trabajo, sería dar contestación a estas dos preguntas:

$1 .^{\circ}$ ¿Cuándo y por qué procesos saben los niños de la existencia de segmentos independientes en el castellano escrito?

$20^{\circ}$ ¿Podrían ser estos conocimientos achacables a la instrucción escolar o, por el contrario, sugieren una conciencia meta-lingüística autónoma fruto de la reflexión que la trasposición escrita de la lengua obliga a hacer?

Según nuestra opinión, y a diferencia de otras nociones más permeables a la observación infantil, los niños no suelen tener ideas espontáneas de estos temas; generalmente es el aprendizaje escolar el iniciador y dosificador de las ideas infantiles al respecto; ahora bien, en caso de que estas primeras opiniones resultasen ciertas, siempre sería interesante conocer los procesos mentales por los que los niños regulan y organizan estos aprendizajes.

Sondear psicológicamente temas meta-lingüísticos, presenta serias dificultades, puesto que el tratamiento exclusivamente verbal —con el lenguaje oral- corre riesgo de deformación al no disponerse de concreción real sobre la que dirigir la atención infantil. La utilización del código lecto-escrito elimina estos inconvenientes, si bien dirige la actividad infantil hacia las unidades relevantes para esta modalidad, presentando además series dudas (Smith, '1973; Ferreiro, E., y Teberosky, A., 1979) de ser generalizado a las formas orales.

Conscientes de estos problemas optamos por investigar las posibilidades de segmentación del castellano con material lecto-escrito, ya que las objecciones metodológicas y teóricas eran más fielmente objetivables. De esta forma lo que sigue responde a las posibilidades de separación-segmentación de sujetos españoles en su idioma escrito.

\section{SITUACION EXPERIMENTAL}

El material utilizado en las sesiones experimentales fueron tres textos creados (escritos) por el propio sujeto experimental en una prueba previa a la de segmentación. La técnica experimental fue la siguiente: en primer lugar, en una situación de relación individual, se pidió a cada sujeto la escritura (ante una cuartilla completamente en blanco) de: «una historia corta, lo más corta posible» sobre dos objetos que el experimentador dejaba unos segundos sobre la mesa y eran retirados y sustituidos por otros (hasta tres veces) una vez el niño había escrito un texto. La consigna se repitió, pues, tres veces sobre :tres parejas de objetos diferentes, a saber:

- Obj. Animado-persona: «un muñeco». Obj. Inanimado: «un lápiz».

- Obj. Animado-persona: «una muñeca». Obj. Animado-animal: "un perro".

- Obj. Animado-animal: «un elefante». Obj. Inanimado: «un plato".

Los objetos eran miniaturas de unos diez centímetros de altura. Se admitieron todo tipo de textos-resultado; si bien, si eran demasiado largos o incluían introductores de narración, el experimentador sugería 
su reducción mediante expresiones del tipo: «¿Es la historia más corta que puedes hacer?» «¿No podrías hacerla más corta?» Tras estas sugerencias los textos se aceptaban como material para la segmentación.

Una vez escritos los tres textos, el experimentador proporcionaba al niño unas tijeras y expresaba la orden siguiente: «Separa las historias en trozos. Puedes hacerlo como quieras, separar por donde quieras, pero fíjate bien cómo lo haces porque después me has de explicar por qué lo has hecho así.» No se daba ninguna explicación adicional, únicamente se recordaba la necesidad de explicar posteriormente las razones de la separación. Una vez efectuado el recorte se interrogaba al niño sobre el por qué de su separación y sobre la posibilidad de realizar trozos diferentes, más pequeños o más grandes. Finalmente se tuvo especial cuidado en no utilizar ninguna palabra de referencia a las unidades buscadas («historia» y «trozo» fueron las habituales).

\section{Población}

Compuesta por 88 niños de un centro escolar de EGB estatal. El sistema de enseñanza para la lecto-escritura seguido por todos ellos fue el tradicional (letras-sílabas-palabras, dictados y copias). Su distribución por edades y sexo fue la siguiente: Entre 5; 6 y 10; 11 años, a razón de 16 sujetos por año. Aproximadamente la distribución por sexos era del 50 por 100 (aunque no siempre, por razones de organización escolar). Entre $5 ; 6$ y $5 ; 11$, el número de sujetos fue de ocho.

\section{Resultados}

Se registraron dos diferentes momentos evolutivos cuya linea fronteriza estaría situada aproximadamente en los siete-ocho años, que dividiría a la población en dos sectores claramente diferenciados ${ }^{1}$.

1. El primero correspondiente al momento evolutivo I (ver cuadro adjunto), formado por los sujetos más jóvenes de nuestra muestra, cuya principal característica estaría en la progresiva búsqueda de las unidades ortográficamente correctas (las palabras tradicionales) y, por tanto, su tendencia a separar por los espacios en blanco que dejan entre las unidades.

TABLA I.

Porcentaje de resultados

\begin{tabular}{|c|c|c|c|c|c|c|}
\hline \multirow[t]{3}{*}{ Edad } & \multirow[t]{3}{*}{0} & \multicolumn{4}{|c|}{1} & II \\
\hline & & \multicolumn{3}{|c|}{ IA } & \multirow[t]{2}{*}{18} & \\
\hline & & let & sil & $\rightarrow$ pal & & \\
\hline $\begin{array}{c}5 ; 6 \\
6 \\
7 \\
8 \\
9 \\
10\end{array}$ & $\begin{array}{l}12,50 \\
6,25 \\
= \\
= \\
-\end{array}$ & $\begin{array}{l}25,0 \\
= \\
= \\
=\end{array}$ & $\begin{array}{c}12,50 \\
6,25 \\
= \\
=\end{array}$ & $\begin{array}{c}50 \\
43,75 \\
18,75 \\
12,50 \\
- \\
-\end{array}$ & $\begin{array}{c}\overline{43,75} \\
62,50 \\
50,00 \\
68,75 \\
43,75\end{array}$ & $\begin{array}{c}- \\
\overline{18,75} \\
37,50 \\
31,25 \\
56,25\end{array}$ \\
\hline
\end{tabular}

Tipos de secciones: $O=$ separaciones arbitrarias. $1=$ separaciones por los espacios reales entre grafias. $\mathrm{IA}=$ separaciones hacia las palabras. $\mathrm{IB}=$ separaciones palabra. II = separaciones de más de una palabra. 
Tal como se observa en el cuadro hay sujetos de todas las edades en este momento. Los datos no apoyan una evolución genética, sino por el contrario una influencia escolar muy marcada. Es por esto que sólo los sujetos de cinco y seis años cronológicos ofrecen conductas de separación en letras y sílabas, debido con toda seguridad a que su aprendizaje del lenguaje escrito escolar está fomentando la utilización cognoscitiva de los elementos en este sentido; después, el niño separa palabra a palabra o intenta buscar las unidades-palabra consustanciales con el código escrito.

El niño tiene hasta los ocho años ideas confusas de lo que son las unidades-palabra del castellano con corrección ortográfica; existe hasta esta edad una progresiva adecuación entre dos polos atrayentes: el valor semántico y los espacios ortográficos.

El sujeto de cinco a ocho años (todavía un 12,50 por 100 a esta edad) procede a separar por espacios, pero lo escrito entre ellos corresponde a unidades significativamente correctas no ortográficamente. Así es frecuente encontrar grupos significativos donde los elementos sin significación propia se agrupan a los que la poseen. Grupos tales como:

\begin{tabular}{lll} 
aviaunavez/ & por & había una vez ${ }^{2}$ \\
superrito/ & por & su perrito \\
cepaseaba/ & por & que paseaba \\
tebebiste/ & por & te bebiste \\
& \multicolumn{2}{c}{...etc... }
\end{tabular}

Ejemplos como los que anteceden son numerosos, corresponden a conductas integradas en el grupo (IA $\rightarrow$ pal) del cuadro adjunto. En el apéndice se muestran ejemplos de los recogidos. El niño está preocupado por separar de la mejor manera posible, pero el valor significativo de sus unidades no es plenamente coincidente con el valor ortográfico-adulto.

La escuela busca y valora las separaciones ortográficas y únicamente prestando atención al comportamiento del niño se descubre, en errores como los señalados, la verdad sobre los conflictos por los que evolutivamente pasa, las adaptaciones que hace hacia un códigosistema convencionalmente estructurado por el mundo adulto al cual el proceso educativo le lleva. Mediante estas dificultades, que se detectan en la prueba por la necesidad en que se encuentran de seccionar con unidades un texto escrito, los niños nos descubren las posibilidades de su pensamiento y el nivel de comprensión que de estas unidades poseen.

Este proceso de adaptación, influenciado en grado sumo por la enseñanza escolar, se produce, según nuestra población, hasta los siete-ocho años y según un proceso pendular que se hace muy sospechoso de instrumentalismo escolar. El tal proceso pendular, se da porque los fenómenos de mala separación están representados fundamentalmente por dos conductas contradictorias, cuyo equilibrio conlleva la correcta separación según las normas del castellano. La primera de éstas empuja a los niños a separar según la significación a nivel de la lógica de su pensamiento; de esta forma los niños no consideran independientes gran número de morfemas que lo son para el castellano, tales como: la separación entre artículos y sustantivos, las dife- 
rentes composiciones de los verbos compuestos, las flexiones de algunos tiempos, los verbos y las preposiciones que los modifican, etc.

La escuela produce a la vez, simultáneamente, el otro proceso contradictorio que señalaba, porque en lugar de respetar el pensamiento del niño y su proceso de «ir haeia» las unidades adultas por diferenciación de significados, se limita a corregir y censurar sin ni siquiera justificar sus actuaciones $y$ entonces se dan en los niños fenómenos de excesiva separación (también incluidos en IA $\rightarrow$ pal), tales como:

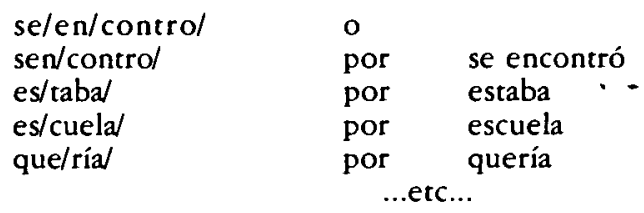

La causa parece estar en que tanto /en/, como /es/, como /que/, pueden aparecer como palabras solas y el niño en un exceso de acomodación o por mal aprendizaje, generaliza casos sin tener una buena comprensión de los fenómenos. (En el apéndice sẹj recogen ejemplos de estos comportamientos.)

Finalmente, aproximadamente a los siete años ya se da lä perfecta adecuación (grupo IB) entre las conductas recogidas y las normas ortográficas de separación en palabras.

Es característico también en los menores de ocho años ante los sondeos de justificación, el no encontrar otras posibilidades de separar más que la que han hecho; aseguran que «no estaría bien» ‘cortar en rrozos más pequeñós o más grânúdes; ảgunosos, entreven posibilidades físicas de hacerlo, pero confirman que sería irrelevante. Las razones oscilan desde: «me han enseñado así», "es así mejor", hasta las: "ya se sabe que las palabras van solas", «he cortado palabra a palabra/la/ es una palabra y /niña/ es otra palabra no las puedo poner juntas». Las unidades más pequeñas (letra, sobre todo) son consideradas como posibles, pero menos buenas que las secciones-palabra.

2. El segundo grupo, que hemos clasificado como II (del cual ya se encuentran algunos ejemplos a siete años), estaría caracterizado por la consideración de unidades más grandes, de varias palabras, olvidando las separaciones ortográficas y superándolas en base a considerar las posibles relaciones sintácticas entre los elementos.

Las conductas de recortado no están aquí en correspondencia con los espacios vacíos entre las palabras, éstas aparecen en secuencias correctas, pero el niño prescinde de estos espacios buscando unidades superiores formadas por varias palabras que en su explicación relaciona. Estos niños tienen «in mente» la unidad general de la frase, al menos de algunos elementos dentro de la frase y además ninguno de ellos desprecia la separación palabra-palabra; en los sondeos posteriores al recortado, todos reconocen que la separación propia de I-B es una posibilidad, pero espontáneamente en la experiencia agrupan según unidades que superan la morfología analítica.

En principio, sólo algunos elementos se agrupan: Y con el lápiz/, /el lobo! a! se escribe! muy semejantes a lac agrupaciones de (IA $\rightarrow$ Dal). pero con la importante diferencia de que estos niños tienệ clara la 
buena separación ortográfica y saben que cada uno de los elementos son unidades diferentes. Así pues, jerárquicamente existe una categoría superior que une estas unidades y que no es otra que la relación sintáctica. Posteriormente con la edad, las agrupaciones se hacen más semejantes a las recogidas por las sintaxis adultas.

Aunque actualmente los gramáticos prefieren organizar frases del tipo que recogemos, según la estructura bipartita de sintagma nominal-sintagma verbal, con las consiguientes divisiones posteriores; en las conductas espontáneas de los niños se reproduce muy poco este modelo, únicamente en un 4,30 por 100 de los casos. El modelo más frecuente es el de tripartición, según la estructura de S-V-O o S-V-Adv., empleada en un 59,3 por 100 de los casos. Se encuentran, asimismo, estructuras bipartitas no recogidas por las gramáticas, del tipo (SV)-O (8,7 por 100).

Los porcentajes de todas las conductas recogidas en II son:

\begin{tabular}{|c|c|}
\hline & $\%$ \\
\hline Elementos inmediatos-aislados $\ldots \ldots \ldots$ & 21,7 \\
\hline $\begin{array}{l}\text { Tripartita: S-V-O y elementos adverbiales } \\
\text { si los hay } \ldots \ldots \ldots \ldots \ldots \ldots \ldots \ldots \ldots \ldots \ldots\end{array}$ & 59,3 \\
\hline Bipartita FN-FV .. & 4,3 \\
\hline Bipartita SV-O o S-VO & 8,7 \\
\hline $\begin{array}{l}\text { Ambas (bipar. trip.) porque la frase original es } \\
\text { más complicada } \ldots \ldots \ldots \ldots \ldots \ldots \ldots \ldots \ldots \ldots\end{array}$ & 6,0 \\
\hline
\end{tabular}

Las explicaciones infantiles de justificación en esta etapa señalan la relación entre elementos tanto si únicamente hacen referencia a componentes muy inmediatos (artículo y sustantivo, por ejemplo), como si otras unidades no tan relacionadas se aíslan juntas en franca separación de otros elementos de la frase.

La verbalización de las razones es a veces ambigua, por ejemplo, un sujeto de 7;4 (su trabajo está recogido en el apéndice) explica lo siguiente:

-Ex: ¿Cómo has cortado?

-R: "Así... (señala) éstos (/un lápiz/) van juntos porque son de la misma frase.»

-Ex: ¿Cómo estaría si cortases de una en una?

-R: «Un poco peor, porque hay palabras que van juntas porque si no, no irian juntas /un elefante/, /un plato/, /habia una vez/ han de ir juntas, son de la misma frase."

Las justificaciones de este sujeto nos permite detectar «esa noción" jerárquicamente superior a la palabra. El confusionismo que manifiesta con las denominaciones (bastante común en edades superiores a los siete años) nos demuestra en qué medida hay disociación entre las primeras nociones reflexivas y los aprendizajes escolares.

En otros casos, se da en el niño una comprensión muy cercana a las relaciones gramaticales subyacentes si bien muy intuitiva; a medida que aumenta la edad, razones más específicas se añaden a los «esto es un poco junto" o "están juntas porque quedan mejor". Por ejemplo, G. H. de 10;9 años (véase su protocolo en el apéndice), dice:

«En [la niña desconfía del lobol, he cortado por /la niña/, porque después/desconfial y después/del lobo/; / la niña/ nos explica qué es; desconfial que nos indica como lo que teme... quiere decir que tiene miedo, y/del lobo/ que nos indica de quién. En lun hombre con un lápiz puede escribir], es correcta así, porque /puede escribir/ puede 
ir solo porque nos indica lo que puede hacer alguien. La última a mí me parece que asi queda más correcta porque ningún elefante come en el plato."

-Ex: ¿Cómo te parece que quedaría si separaras de una en una?

$-\mathrm{R}$ : “QQué quedaran palabras sueltas?»

-Ex: Sí.

-R: "Sí, pero yo creo que peor porque no quedarian bien tantas palabras sueltas, no tendrían sentido."

Nuestros sujetos de II, usan razones fundamentalmente significativas para justificar las relaciones entre las palabras, sólo de esta forma pueden entenderse las utilidades pragmáticas que los elementos unidos colaboran a prestar a nuevas posibles frases en opinión de sujetos como G. H.

\section{CONCLUSIONES}

Los resultados descritos demuestran desde nuestra óptica dos tipos de conclusiones:

1. Que el proceso de comprensión metalingüístico de las unidades del lenguaje escrito y de sus relaciones es muy lento, realizándose un nuevo proceso de construcción (diferente al del idioma oral) que lleva desde el seguir las pautas marcadas por el bien hacer escolar hasta cuestionarse la utilidad y el valor aislado o agrupado de los diversos elementos del idioma.

Se puede decir que los sujetos del estadio II, estarían cognoscitivamente en condiciones de comprender las diferentes funciones entre palabras y el manejo de grupos de unidades en función de elementos morfo-sintácticos englobadores. Los sujetos del estadio IB, han superado fases de construcción ortográfica, pero sin embargo no se cuestionan la existencia de unidades superordenadas.

Los sujetos de IA-pal son conscientes únicamente del régimen de dependencia de las palabras significativas y no significativas respecto de los espacios ortográficos. Si el idioma presenta una inadecuación o una arbitrariedad, el niño duda entre las aplicaciones semánticas y las convencionales. Las metas convencionales son especialmente fuertes durante los 7, 8 y 9 años (mayoria de separación IB); sólo a partir de 10 años los porcentajes de niños capaces de plantearse y de simultanear varios tipos de segmentaciones supera el 50 por 100 .

2. Que la escuela interviene de una manera decisiva en los resultados que ofrecen los niños. La escritura es algo marcadamente escolar y desgraciadamente las ya importantes investigaciones psicológicocognitivas sobre los procesos espontáneos en estos temas (Smith, F., 1973, y Ferreiro-Treberosky, 1979, 1981, entre nosotros) todavía no han recibido contestación ni aplicación escolar.

Día a día se está demostrando cómo el niño que realiza los aprendizajes de la versión lecto-escrita de su idioma materno tiene unas estructuras cognitivas que aplica activamente a este proceso de conocimiento (no se trata únicamente de niveles perceptivos o psicomotores). El largo proceso constructivo de más de cuatro años de duración ànte una senciila prueda de segmentación contribuye, a nüesiru parecer, a demostrarlo. 


\section{Notas}

1 Se encontraron algunas respuestas de segmentaciones no lingüisticas (momento 0 del cuadro adjunto), se trataba de separaciones arbitrarias detectadas antes de siete años. Por ejemplo, S. R. de 6;0; recorta las grafías por el medio, pero los grupos-resultado no se identifican con ninguna unidad. Se exṕresa asi:

Ex: ¿Cómo has cortado? -R: «así... se puede hacer como un rompecabezas... estas (señala/quiere/) las tendré que cortar juntas porque están pegadicas".

Ex: ¿En qué te has fijado para cortar? R: - «como un rompecabezas".

2 Las barras indicarán en todos los ejemplos las separaciones reales que hacen los sujetos en el recortado de las frases.

\section{Referencias}

BRown, A. L., y SMILEY, S. S.: «Rating the importance of structure units of prose passages: A problem of metacognitive development». Child Development, 1977, 48, 1-8.

Ferreiro, A., y Teberosky, A.: Los sistemas de escritura en el desarrollo del niño. México. Siglo XXI. 1979. MCCAugheY, M-Juola, J. F.; SChadier, M., y WARD, N.: «Whole-Word units are used before orthographic knowledge in perceptual development". Journal of Experimental Psych, 1980, 30, 41.1-421.

JOHNSON: «Recall of prose as a function of the structural importance of the linguistic units". Journal of Verbal Learning and Verbal Behavior, 1970, 9, 12-20.

SMITH, F.: Psychololinguistics and reading. Holt, Rinehart and Wiston. 1973.

VYGoTSKI, L S.: El desarrollo de los procesos psicológicos superiores. Grijalbo, 1979.

Varios: «Aprenentage de la lectura i l'escriptura». Seminari organizat per l'ICE. Universitat Autónoma de Barcelona.

\section{Apéndice}

Cad 5.6.5.

$M, 7$; 5;7 avios.
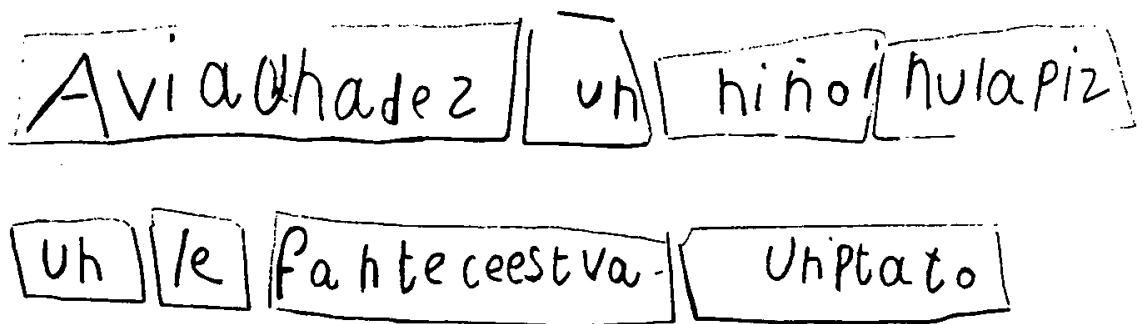

Cod: 6.6 .3

Nive: 5.C. ethed: 6'10
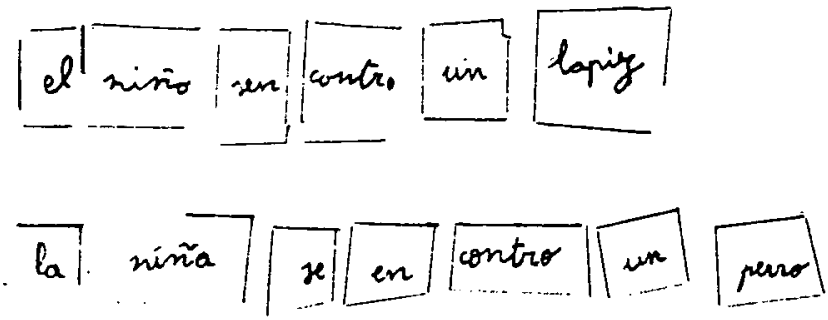

Codigo: 1.0 .3

nombre: T.G; $7.3 \mathrm{ancs}$

el mino y lacala is cuela $y$ yedir 


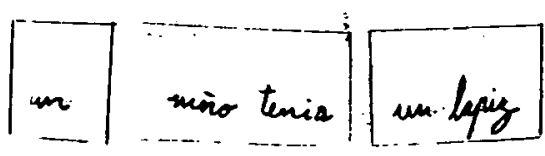

J. 6 , edicil : 7.4

avia una ve unanivia que se encontro unlovo

im elefante Ltenia im plato

Cody: 9.68

Nom: J.P; edod: $9: 9$

\&l niño escribe / convel lapir.

La miña | pasea con superizo

Lel elofante come $\mid$ en el ptato

Cod: 10.6 .8

Nom: G. H; edad: $40^{\prime 9}$

Un trombire con un lapise prede eservibir

dá niña desconfia Jel lobo.

Ee elefante no comesen plato / camo los hombres.

Resumen

Mediante tareas de segmentación de textos, se investiga la evolución infantil en el conocimiento de las unidades

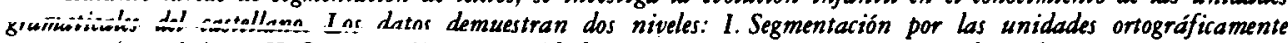
correctas (espacios), y II. Segmentación por unidades superiores teniendo en cuenta ias réuciuraci sirisiónititios. S. argumenta sobre la influencia escolar en la solución de estas tareas.

85 
Through text segmentation task, this paper shows the development of children in the knowledge of the grammatical units in spanish. Results show two-levels: I. Segmentation by the correct ortographic units (spaces), and II. Segmentation by the superior units taking into account syntactic relationship. There is discussion about the educational influence in the solving of these tasks.

\section{Résumé}

La recherche de l'évolution (développment) de l'enfant sur la reconnaissance des unités grammaticales espagnoles est faite par des tâches de segmentation de textes. Les resultats demontrent deux niveaux: I. La segmentation par les unités ortbographiquement correctes (espaces), et II. La segmentation par les uniés supérieures; on rend compte des relations syntactiques. On fait une argumentation au sujet de linfluence de l'école à la réussite de cettes tâches. 